\title{
Interaction of two fluxons near a local inhomogeneity in a long Josephson junction
}

\section{Malomed, Boris A.; Nepomnyashchy, Alexander A.}

\section{Published in:}

Physical Review B

Link to article, DOI:

10.1103/PhysRevB.45.12435

Publication date:

1992

Document Version

Publisher's PDF, also known as Version of record

Link back to DTU Orbit

Citation (APA):

Malomed, B. A., \& Nepomnyashchy, A. A. (1992). Interaction of two fluxons near a local inhomogeneity in a long Josephson junction. Physical Review B, 45(21), 12435-12441. https://doi.org/10.1103/PhysRevB.45.12435

\section{General rights}

Copyright and moral rights for the publications made accessible in the public portal are retained by the authors and/or other copyright owners and it is a condition of accessing publications that users recognise and abide by the legal requirements associated with these rights.

- Users may download and print one copy of any publication from the public portal for the purpose of private study or research.

- You may not further distribute the material or use it for any profit-making activity or commercial gain

- You may freely distribute the URL identifying the publication in the public portal

If you believe that this document breaches copyright please contact us providing details, and we will remove access to the work immediately and investigate your claim 


\title{
Interaction of two fluxons near a local inhomogeneity in a long Josephson junction
}

\author{
Boris A. Malomed* \\ Physics Laboratory I, Technical University of Denmark, DK-2800 Lyngby, Denmark \\ and P. P. Shirshov Institute for Oceanology, U.S.S.R. Academy of Science, 23 Krasikov Street, Moscow 117259, U.S.S.R.
}

Alexander A. Nepomnyashchy ${ }^{\dagger}$

Department of Mathematics, Technion-Israel Institute of Technology, Haifa 32000, Israel

(Received 8 July 1991)

\begin{abstract}
Collision of a free fluxon in a long dc-driven damped Josephson junction with a fluxon pinned by the microshort or microresistor is analyzed by means of perturbation theory. It is demonstrated that, in the case of like polarities of the fluxons, three different outcomes of the collision are possible: depinning, capture, and exchange. If the polarities are opposite, the possible outcomes are depinning, annihilation, and a quasielastic collision. The boundaries between the different regimes in the parametric space of the model are found.
\end{abstract}

\section{INTRODUCTION}

In this work we develop an analysis of collisions between a fluxon (magnetic-flux quantum) pinned by a local inhomogeneity in a dc-biased damped long Josephson junction ( $\mathbf{L J J}$ ), and a free fluxon. This process is an interesting physical problem, which may also have some practical importance in the designing of elementary logic units for the Josephson computer. Besides, the outcome of the collisions between free and pinned fluxons is important in determining the $I-V$ (current-voltage) characteristic of a very long junction, containing a regular or random lattice of microinhomogeneities and a "rarefied gas" of fluxons.

The analysis of the problem is based on the well-known perturbed sine-Gordon (SG) equation, ${ }^{1}$

$$
\phi_{t t}-\phi_{x x}+\sin \phi=-f-\gamma \phi_{t}+\epsilon \delta(x) \sin \phi,
$$

where $\phi$ is the dimensionless magnetic flux, $f$ is the bias current density, and $\gamma$ is a dissipation coefficient. The last term on the right-hand side of (1.1) corresponds to a local inhomogeneity of the maximum Josephson current density (a microshort and a microresistor for negative and positive $\epsilon$, respectively).

The interaction between a fluxon and localized inhomogeneities was studied experimentally in Refs. 2 and 3. The experimental results support the theoretical model (1.1).

In the absence of perturbations, i.e., at $f=\gamma=\epsilon=0$, the fluxon is described by the well-known kink solution of the $\mathrm{SG}$ equation,

$$
\phi_{k}(x, t)=4 \tan ^{-1}\left[\exp \left[\sigma \frac{x-\xi(t)}{\left(1-v^{2}\right)^{1 / 2}}\right]\right],
$$

where $\sigma= \pm 1$ is the polarity of the fluxon, $\xi(t)=v t+\xi_{0}$ is the coordinate of its center, and $v$ is its velocity $\left(v^{2}<1\right)$. In the homogeneous dc-biased damped LJJ $(\epsilon=0$; $f, \gamma<<1)$, the fluxon's velocity $v_{0}$ is uniquely determined by the balance between the driving and friction forces, ${ }^{1}$

$$
v_{0}=\sigma\left[1+(4 \gamma / \pi f)^{2}\right]^{-1 / 2} \operatorname{sgn} f .
$$

In the "nonrelativistic case" $\left(f<<\gamma, v_{0}^{2}<<1\right)$ Eq. (1.3) simplifies to

$$
v_{0}=\pi \sigma f / 4 \gamma \text {. }
$$

If there is a weak inhomogeneity $(|\epsilon|<<1)$, one can analyze the motion of a fluxon using the perturbation methods. ${ }^{1}$ In the adiabatic approximation (when the radiative losses and a distortion of the fluxon shape are ignored), one can derive the motion equation of a nonrelativistic flux, that proves to be equivalent ${ }^{1}$ to the equation describing the motion of a classical particle with the mass $m=8$ in the potential

$$
U_{\mathrm{eff}}(\xi)=2 \pi \sigma f \xi-2 \epsilon \operatorname{sech}^{2} \xi,
$$

in the presence of the friction force

$$
F_{\mathrm{fr}}=-8 \gamma \frac{d \xi}{d t}
$$

[it is assumed $(d \xi / d t)^{2}<<1$, otherwise the effective particle obeys the "relativistic" equation of motion].

The potential (1.5) can pin a fluxon, provided ${ }^{1}$

$$
f<f_{\mathrm{cr}} \equiv(4 \sqrt{3} / 9 \pi)|\epsilon|
$$

in this case, the potential has two equilibrium positions, one stable and one unstable (the minimum and the maximum of the potential).

If the bias current density lies in the interval $0<f<f_{\text {cr }}$, the LJJ with sparse inhomogeneities may support two types of fluxons: the pinned ones, and the free fluxons moving between the inhomogeneities. If both colliding fluxons have like polarities, the collision between the free and pinned ones may have three different outcomes: (i) the capture of both fluxons; (ii) the "exchange", i.e., the capture of the free fluxon and depinning of the pinned one; and (iii) the depinning of the formerly pinned fluxon without the capture of the free one. In what follows, these three possibilities will be referred to 
as the capture, exchange, and depinning, respectively. For the case of like polarities, the collision is analyzed in detail in Sec. II.

If the free and pinned fluxons have opposite polarities, one can expect the depinning as one possible outcome of the collision. Instead of the exchange, in the case of the opposite polarities one can have the elastic collision, when the pinned fluxon remains pinned, and the free one keeps moving in the same direction. Finally, in this case one can have the annihilation of the fluxon-antifluxon pair instead of the capture in the case of the like polarities. The analysis of the fluxon-antifluxon collision is given in Sec. III.

Finally, in the concluding Sec. IV we briefly discuss possible generalizations of the problem considered in this work.

\section{THE COLLISION BETWEEN THE FLUXONS WITH LIKE POLARITIES}

In this section we will consider the collision between the free and pinned fluxons whose coordinates are $\xi_{1}$ and $\xi_{2}$ (we assume $\xi_{2}<\xi_{1}$ ), and the polarities are $\sigma_{1}=\sigma_{2}=1$. The analysis will be based on the perturbation theory, so that all the parameters $f, \gamma$, and $\epsilon$ in the underlying equation (1.1) will be assumed small. The coordinate $\xi_{0}$ of the pinned fluxon is determined by the equation $d U_{\text {eff }} / d \xi=0$, i.e.,

$$
\sinh \xi_{0} \operatorname{sech}^{3} \xi_{0}+\pi f / 2 \epsilon=0 .
$$

\section{A. The depinning of the pinned fluxon}

First of all we will find critical conditions for the depinning of the pinned kink. The qualitative idea underlying the subsequent analysis is that the fluxon will not be depinned if the kinetic energy transferred to it by the free one is dissipated during the collision. We will see below that, at $f \sim|\epsilon|$, this is possible provided the dissipation is sufficiently strong, $\gamma \gtrsim|\epsilon|^{1 / 2}$. In this case, the motion of the fluxon is nonrelativistic. As it is well known, the fluxons with like polarities repell each other and, if they are nonrelativistic, they stay at a distance much larger than their proper size (which is $\sim 1$ in the notation adopted). In this case, the effective potential of the fluxon-fluxon interaction is ${ }^{4}$

$$
V_{\text {eff }}\left(\xi_{1}-\xi_{2}\right)=32 e^{\xi_{2}-\xi_{1}}
$$

(recall that we assume $\xi_{2}<\xi_{1}$ ). Considering the fluxons as mechanical particles, ${ }^{1}$ and changing the variables

$$
\begin{aligned}
& \tau \equiv\left(\frac{|\epsilon|}{2}\right)^{1 / 2} t, \quad \Gamma \equiv\left(\frac{2}{|\epsilon|}\right)^{1 / 2} \gamma, \\
& F \equiv \frac{\pi f}{2|\epsilon|}, \quad \xi_{1} \equiv \xi_{1}^{\prime}-\ln \left(\frac{|\epsilon|}{8}\right),
\end{aligned}
$$

we obtain the following system of the equations of motion

$$
\begin{aligned}
& \frac{d^{2} \xi_{1}^{\prime}}{d \tau^{2}}+\Gamma \frac{d \xi_{1}^{\prime}}{d \tau}+F-e^{\xi_{2}-\xi_{1}^{\prime}}=0, \\
& \frac{d^{2} \xi_{2}}{d \tau^{2}}+\Gamma \frac{d \xi_{2}}{d \tau}+F+\operatorname{sgn} \epsilon \sinh \xi_{2} \operatorname{sech}^{3} \xi_{2}+4 e^{\xi_{2}-\xi_{1}^{\prime}}=0 .
\end{aligned}
$$

The influence of the inhomogeneity on the motion of the incoming free fluxon can be ignored. In this case, the force generated by the inhomogeneity and acting on the incoming free fluxon is of order $\epsilon^{3}$ and may be neglected. This system has to be solved with the following initial conditions at $\tau \rightarrow-\infty$ :

$$
\begin{aligned}
& \xi_{1}^{\prime} \rightarrow \infty, d \xi_{1}^{\prime} / d \tau=-F / \Gamma ; \\
& \xi_{2}=\xi_{2}^{(0)}, d \xi_{2} / d \tau=0 .
\end{aligned}
$$

The pinned state of the fluxon exists if $F<F_{\text {cr }} \equiv 2 \sqrt{3} / 9 \approx 0.3849$. Adding up the expressions on the left-hand sides of Eqs. (2.4a) and (2.4b), we find the condition necessary for the inhomogeneity to hold both fluxons

$$
-2 F+\operatorname{sgn} \epsilon \sinh \xi_{2} \operatorname{sech}^{3} \xi_{2}=0 .
$$

A solution of (2.6) exists if

$$
F<F_{\text {cr }} / 2 \text {. }
$$

Thus, the boundary of the capture region in the parametric space, $F=F(\Gamma)$ [it is implied that the capture takes place at $F<F(\Gamma)]$, lies beneath the line $F=F_{\text {cr }} / 2$.

To proceed with the analysis, let us first consider the repulsive inhomogeneity $(\epsilon<0)$. In the limit $F, \Gamma \rightarrow 0$, it is possible to find the asymptotics of $F(\Gamma)$ analytically. In this case, the pinned fluxon rests far from the inhomogeneity:

$$
\xi_{0} \approx \frac{1}{2} \ln (F / 4)
$$

and the influence of the inhomogeneity on the collision is negligible. If the scaled velocity $V \equiv F / \Gamma$ is of order one, then (2.4) in the lowest approximation can be written in the form

$$
\begin{aligned}
& \frac{d^{2} \xi_{1}^{\prime}}{d \tau^{2}}-e^{\xi_{2}-\xi_{1^{\prime}}}=0, \\
& \frac{d^{2} \xi_{2}}{d \tau^{2}}+e^{\xi_{2}-\xi_{1}^{\prime}}=0 .
\end{aligned}
$$

System (2.9) can be integrated directly:

$$
\begin{aligned}
& \xi_{1}^{\prime}=\xi_{0}-2 \ln V-V \tau+\ln \left(1+e^{V \tau}\right), \\
& \xi_{1}=\xi_{0}-\ln \left(1+e^{V \tau}\right) .
\end{aligned}
$$

The point $\tau=0$ is the instant at which the two fluxons are at a minimum distance. Certainly the solution (2.10), as well, can be obtained from the exact two-kink solution of the SG equation. Using the expression (2.8), we find that the second (formerly pinned) fluxon gets over the potential barrier $\left(\xi_{2}=0\right)$ at the moment 


$$
\tau_{*}=\frac{1}{2 V} \ln \left(\frac{4}{F}\right) .
$$

Due to the collision, it acquires the velocity

$$
\frac{d \tau}{d \xi_{2}}=V\left(-1+\frac{1}{2} F^{1 / 2}\right) \text {. }
$$

The change of the fluxon's velocity under the action of the force $F$ and the dissipation is small, and we ignore it. The depinning boundary is determined by equating the kinetic and potential energies, which leads to the following expression for the critical velocity:

$$
V=\frac{F}{\Gamma}=1+\frac{1}{2} F^{1 / 2}+\cdots
$$

or

$$
F=\Gamma\left(1+\frac{1}{2} \Gamma^{1 / 2}+\cdots\right) .
$$

In the case of the attractive inhomogeneity $(\epsilon>0)$, the energy of interaction between the fluxons is small at the moment of passing the potential barrier. The energy transferred to the pinned fluxon must be less than the kinetic energy of the incoming one, hence $\lim _{F \rightarrow 0} V(F)>1$. Numerical integration of the system (2.4) with $F=\Gamma=0$ yields $V(0) \equiv V_{\text {cr }}=1.17$.

In the general case (finite $F$ and $\Gamma$ ), Eqs. (2.4) and (2.5) were solved numerically. The results of the calculations are shown in Figs. 1 and 2 (the curves between the regions II and III). The boundary of the capture region is similar to that for the solitary fluxon, ${ }^{5}$ but its horizontal fragment is situated at $F=F_{\mathrm{cr}} / 2$. In the region below this boundary, the inhomogeneity holds back both fluxons; above this boundary the previously trapped fluxon gets depinned.

The dependence of the critical velocity $V=F / G$ and of the critical kinetic energy $E=V^{2} / 2$ of the incoming free

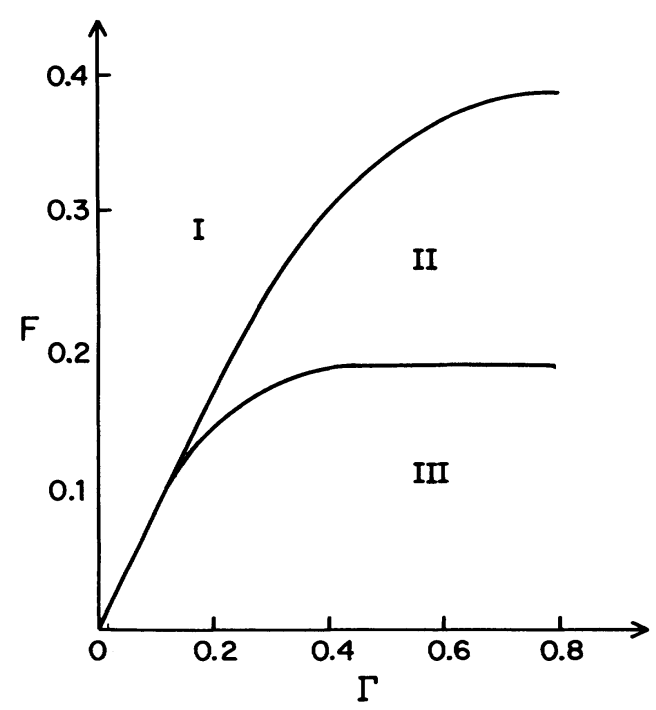

FIG. 1. Interaction regimes in the case of the nonrelativistic incoming fluxon and repulsive inhomogeneity (I-depinning, II-exchange, and III - capture).

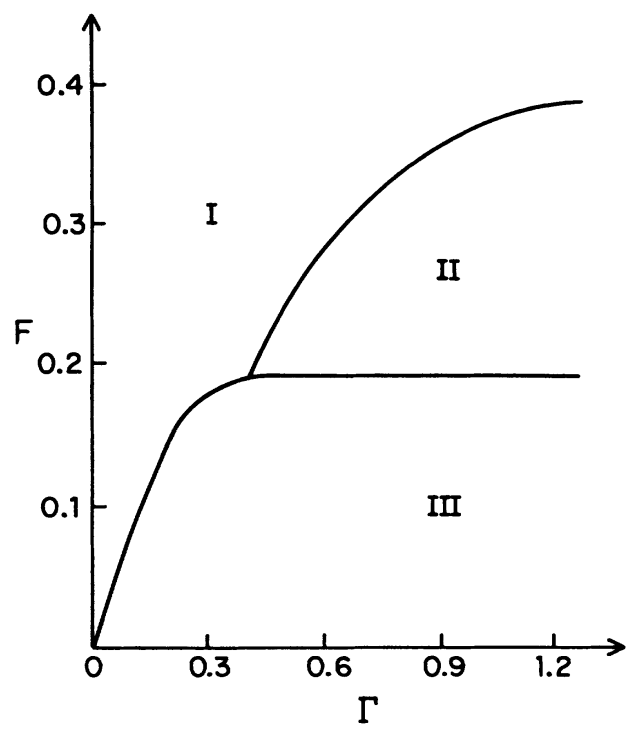

FIG. 2. Interaction regimes in the case of the nonrelativistic incoming fluxon and attractive inhomogeneity (I-depinning, II-exchange, and III-capture).

fluxon on the external force $F$ are shown in Figs. 3 and 4. For comparison, the barrier's height $\Delta \equiv U_{\max }-U_{\min }$ for the potential

$$
U=F \xi+\frac{1}{2} \operatorname{sgn} \epsilon \operatorname{sech}^{2} \xi,
$$

is depicted in Fig. 4. One can see that the threshold value of the kinetic energy $E$ is of the same order as the barrier's height $\Delta$, but it is usually slightly higher than $\Delta$ because of the incomplete energy transfer in the collision. However, for $F$ near $F_{\text {cr }} / 2$ we have $E<\Delta$. This result is not in contradiction to the energy conservation. Actual-

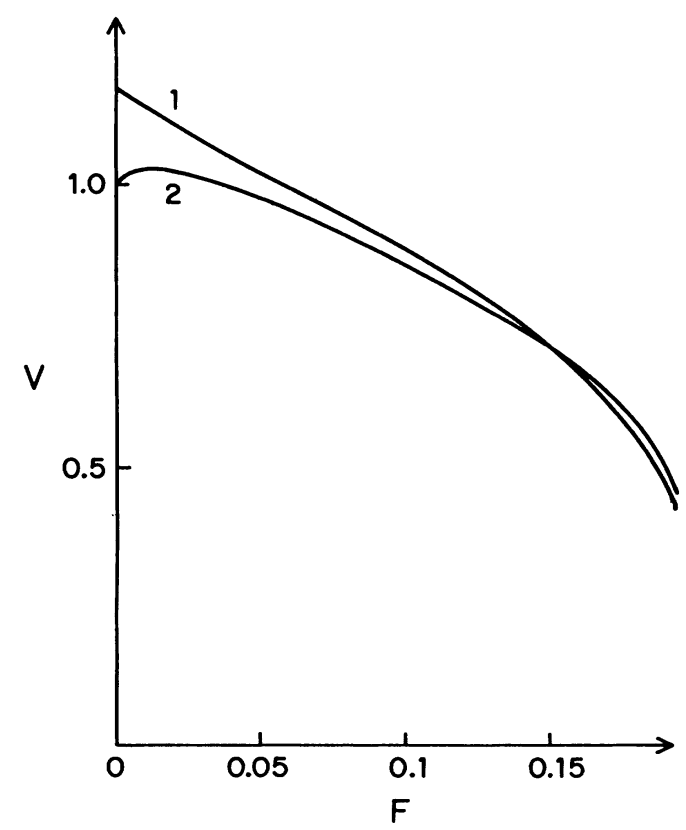

FIG. 3. The critical capture velocity for the attractive (curve 1) and repulsive (curve 2) inhomogeneities. 


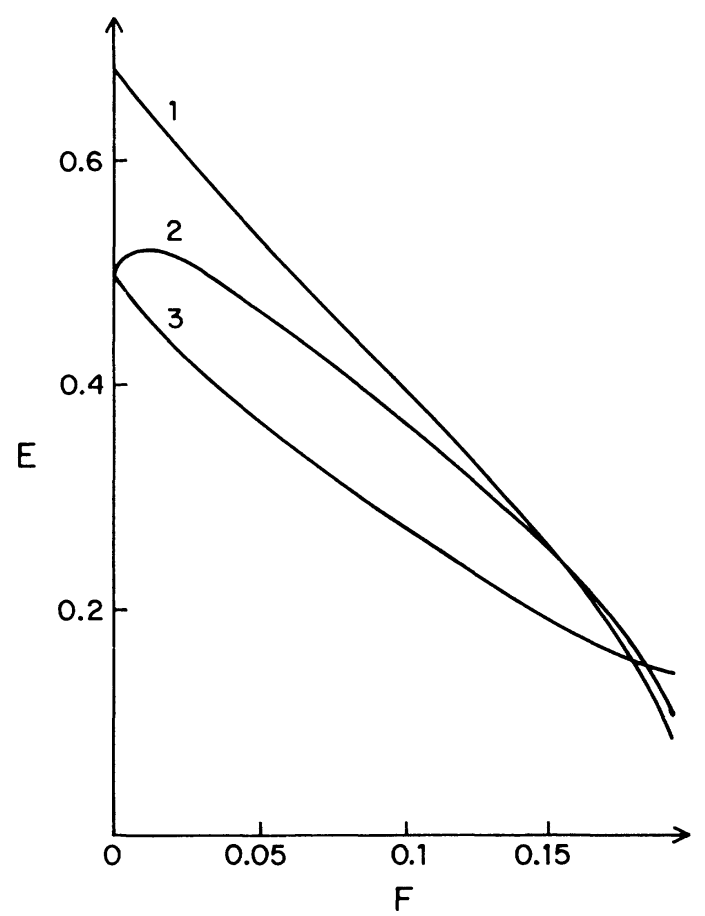

FIG. 4. The critical kinetic energy of the incoming fluxon for the attractive (curve 1) and repulsive (curve 2) inhomogeneities in comparison with the barrier height (curve 3).

$1 y$, the quasistationary balance between the external drive and the dissipation for the incoming fluxon is violated because it is braked, and the force $F$ brings some additional energy into the system.

\section{B. The exchange}

The depinned fluxon leaves the neighborhood of the inhomogeneity during the time of order one and, in what follows, the motion of the fluxon is governed by the equations

$$
\begin{aligned}
& \frac{d^{2} \xi_{1}^{\prime}}{d \tau^{2}}+\Gamma \frac{d \xi_{1}^{\prime}}{d \tau}+F-e^{\xi_{2}-\xi_{1}^{\prime}}=0, \\
& \frac{d^{2} \xi_{2}}{d \tau^{2}}+\Gamma \frac{d \xi_{2}}{d \tau}+F+e^{\xi_{2}-\xi_{1}^{\prime}}=0,
\end{aligned}
$$

until the incoming (initially free) fluxon approaches the inhomogeneity. Then it can be captured by the inhomogeneity (the exchange) or it remains free (the "depinning"').

The crucial factor is the value of the velocity of the fluxon when it is approaching the inhomogeneity. If $F$ and $\Gamma$ are of order one, the fluxon has enough time to retrieve the equilibrium velocity equal to $-F / \Gamma$ [Eq. (1.4)] because the relaxation time for the velocity is $\Gamma^{-1} \sim 1$, and the time of motion is $\sim \ln \left(|\epsilon|^{-1}\right) \gg>1$. When passing the barrier, the interaction between the fluxons is immaterial. Indeed, one can derive from (2.12) the equation for the quantity $X=\xi_{1}^{\prime}-\xi_{2}$ :

$$
\frac{d^{2} X}{d \tau^{2}}+\Gamma \frac{d X}{d \tau}-2 e^{-X}=0 ;
$$

at the moment of the depinning of the formerly pinned fluxon $X \sim 1$. For large $\tau$, the motion is described by the equation

$$
\Gamma \frac{d X}{d \tau}-2 e^{-X}=0
$$

thus, $X \sim \ln (2 \tau / \Gamma)$. When the incoming fluxon approaches the inhomogeneity, the interaction force

$$
e^{-X} \sim \tau^{-1} \sim|\ln | \epsilon||^{-1}<<1
$$

is small in comparison with terms proportional to $F$ and $\Gamma$. So, the motion of this fluxon is fully similar to the motion of the solitary fluxon interacting with the inhomogeneity. ${ }^{5}$ The boundaries between the exchange and depinning regions are shown for this case in Figs. 1 and 2.

The case of small $\Gamma$ needs a special examination. We will consider the cases of repulsive and attractive inhomogeneities separately.

\section{The repulsive inhomogeneity}

Let us assume $F / \Gamma \sim V \sim 1$. In the case of small $F$ and $\Gamma$, the collision of particles is governed by (2.9), while the interaction force is large in comparison with the omitted terms proportional to $F$ and $\Gamma$. One can see [cf. (2.10)] that the term proportional to $F$ becomes important at $\tau \sim|\ln F|$, when the velocity of the incoming fluxon is $\sim F$. Then the fluxon is accelerated by the force $F$.

If $F, \Gamma \gg|\ln | \epsilon||^{-1}$, the fluxon has enough time to attain the equilibrium velocity. The interaction force between the fluxons is of order of $\Gamma|\ln | \epsilon||^{-1}$ [because of $X \sim \ln (2 \tau / \Gamma)]$ and is negligible. So, the capture boundary for the incoming fluxon is the same as for the solitary fluxon: ${ }^{5} \quad F=\Gamma-\Gamma^{2} \ln 2+\cdots$. Comparison with (2.11) shows that the exchange is impossible in this case.

If $F, \Gamma \lesssim|\ln | \epsilon||^{-1}$, the fluxon has not enough time to attain the equilibrium velocity. The interaction between fluxons is, however, weak. Let us introduce the new variables

$$
\begin{aligned}
& F=F_{1}|\ln | \epsilon||^{-1}, \quad \Gamma=\Gamma_{1}|\ln | \epsilon||^{-1}, \\
& \tau=T|\ln | \epsilon||, \quad \xi_{1}=\Xi|\ln | \epsilon|| .
\end{aligned}
$$

We obtain the Cauchy problem for the motion of the fluxon

$\frac{d^{2} \Xi}{d T^{2}}+\Gamma_{1} \frac{d \Xi}{d T}+F_{1}=0, \quad \Xi(0)=1, \quad \frac{d \Xi}{d T}(0)=0$,

that has the solution

$$
\begin{aligned}
& \Xi(T)=1-\frac{F_{1}}{\Gamma_{1}} T+\frac{F_{1}}{\Gamma_{1}^{2}}\left(1-e^{-\Gamma_{1} T}\right), \\
& \frac{d \Xi}{d T}(T)=-\frac{F_{1}}{\Gamma_{1}}\left(1-e^{-\Gamma_{1} T}\right) .
\end{aligned}
$$

The capture boundary for the incoming fluxon is determined by conditions

$$
\Xi\left(T_{*}\right)=0, \quad \frac{d \Xi}{d T}\left(T_{*}\right)=-1
$$

(the fluxon approaches the inhomogeneity with the 
threshold velocity equal to -1 ); hence, we obtain

$$
\Gamma_{1}=-1-V_{*} \ln \left(1-V_{*}^{-1}\right),
$$

where $V_{*} \equiv F_{1} / \Gamma_{1}$. For large $\Gamma_{1}, V_{*}=1+e^{-\Gamma_{1}}$; for small $\Gamma_{1}, V_{*}=1 /\left(2 \Gamma_{1}\right)$. Thus, for $F, \Gamma \lesssim|\ln | \epsilon||^{-1}$ there arises the exchange region $1<V<V_{*}\left(\Gamma_{1}\right)$ lying between the depinning $\left[V>V_{*}\left(\Gamma_{1}\right)\right]$ and capture $(V<1)$ ones.

For $\Gamma|\ln | \epsilon||<<1$, the dissipation has no influence on the collision of the fluxons. Using the general formulas for the interaction of the SG kinks, ${ }^{6}$ one can find the expression for the shift $\Delta \xi$ of the center of the quiescent kink after the collision with another kink moving with the velocity $v$ :

$$
\Delta \xi=2 \ln \left(\frac{1+\left(1-v^{2}\right)^{1 / 2}}{v}\right) \approx \ln \left[\frac{4}{v^{2}}\right) .
$$

Actually, the formula (2.17) gives the coordinate of the center of the formerly moving kink stopped by the collision. If the potential energy $U_{\text {eff }}\left(\xi_{0}+\Delta \xi\right)$ of the stopped kink is larger than the height of the potential barrier $U_{\text {eff }}\left(\xi_{m}\right)$, the kink will not be trapped. Using the expression for the potential, one can show that the boundary between the exchange and depinning regions is determined by the condition $\Delta x=l$, where $l \equiv \mid \epsilon Z / \pi f$ (we suppose $l \gg 1$ ). Hence,

$$
f=\frac{|\epsilon|}{2 \pi(-\ln v)} .
$$

For $v \sim|\epsilon|^{1 / 2}$ we obtain

$$
f=\frac{|\epsilon|}{\pi|\ln | \epsilon||},
$$

With the further decrease of $\gamma$, the critical value of $f$ again becomes dependent on $\gamma$, but now it grows with the decrease of $\gamma$. The equation (2.18) can be written in the form

$$
-v \ln v=|\epsilon| / 8 \gamma .
$$

For $\gamma \gg \epsilon$, the motion of the fluxon is nonrelativistic, and the depinning threshold is given by the formula

$$
f=\frac{|\epsilon|}{2 \pi \ln (\gamma /|\epsilon|)} .
$$

For $\gamma \sim \epsilon$, the velocity of the incoming fluxon is of order one, the fluxons approach each other up to a distance $\sim 1$, and the consideration of all the stages of the interaction in terms of the equations of motion for the centers of the kinks is impossible. However, the interaction force between fluxons is of order one, and it is large in comparison with that induced by the inhomogeneity. Hence, one can use the formula (2.17) to analyze the collision of the fluxons. After the collision, the velocity of the incoming fluxon reduces up to the values $\sim|\epsilon|$, and so its subsequent motion is nonrelativistic $\left(v \sim|\epsilon|^{1 / 2}\right)$, governed by the equation

$$
\frac{d^{2} \xi_{1}}{d t^{2}}+\frac{\pi}{4} f-\frac{|\epsilon|}{2} \sinh \xi_{1} \operatorname{sech}^{3} \xi_{1}=0
$$

(the term $\gamma d \xi_{1} / d \tau$ is immaterial in the case $\gamma \sim|\epsilon|$ ). So,

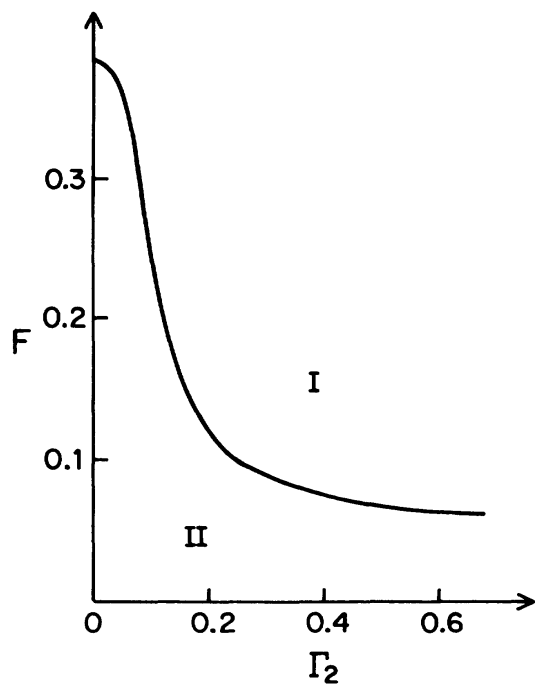

FIG. 5. Interaction regimes in the case of the relativistic incoming fluxon and the repulsive inhomogeneity (I-depinning and II-exchange).

it is enough to compare the potential energy of the stopped kink $U_{\text {eff }}\left(\xi_{0}+\Delta \xi\right)$ and the maximum potential energy $U_{\text {eff }}\left(\xi_{m}\right)$, where $\Delta \xi(v)$ is the shift of the kink's center after the collision, and $\xi_{0}$ and $\xi_{m}$ are the positions of the maximum and minimum of the potential $U_{\text {eff }}(\xi)$. The fragment of the boundary between the exchange and depinning regions, corresponding to this case, is shown in Fig. 5. In Fig. 5 we use the notation $\Gamma_{2} \equiv 2 \gamma /|\epsilon|$. The asymptotic expression at $\gamma \rightarrow 0$ can be written as

$$
\frac{f_{\mathrm{cr}}-f}{|\epsilon|}=\frac{288}{\pi \sqrt{3}}\left(\frac{\gamma}{\epsilon}\right)^{2}
$$

\section{The attractive inhomogeneity}

In the case of the attractive inhomogeneity and $F, \Gamma \rightarrow 0$, the collision between fluxons leads to reflection of the incoming fluxon. The kinetic energy $E_{r}(V) \equiv\left(d \xi_{1} / d \tau\right)^{2} / 2$ of the reflected fluxon has been calculated numerically by solving (2.5) with $\Gamma=F=0$. For $V=V_{c}=1.17, E_{r}=0.184 ;$ for $V \gg 1, E_{r} V^{2}=C=1.1$. Later on, the driving force $F$ makes the fluxon return and, when passing the initial point, it has the velocity $V_{\text {ret }} \sim V_{r}\left[1+O\left(V_{r} / V\right)\right]$.

Let us assume now $\Gamma \rightarrow 0$, and that $V=F / \Gamma \sim 1\left(v \sim \epsilon^{1 / 2}\right)$. In the case $|\ln \epsilon|^{-1}<<\Gamma<1$, the fluxon approaches the inhomogeneity with the velocity $F / \Gamma \sim V_{c}$; in the case $\Gamma \ll|\ln \epsilon|^{-1}$ its velocity has a value lying between $V_{r}$ and $F / \Gamma$, so its energy $E$ is of order one in any case. As the pinning threshold is determined by the condition $E \sim \Gamma$ in the case of the attractive inhomogeneity, the capture is impossible, and there may be no exchange in the case $\Gamma \rightarrow 0, V \sim 1\left(v \sim \epsilon^{1 / 2}\right)$.

However, the exchange region exists if $v \gg \epsilon^{1 / 2}$. We will consider its asymptotic form for $F \rightarrow 0, \Gamma \rightarrow 0$. The capture boundary for the incoming fluxon is determined by the condition 


$$
E_{r}+U_{\mathrm{eff}}(\Delta \xi)=0,
$$

where $E_{r}=8 \cdot(d \xi / d t)^{2} / 2$, since in the case $\Gamma \rightarrow 0$ the reflected fluxon comes back with the same energy. Using the original units for the velocity and energy corresponding to $(2.3)$, we obtain

$$
v=(4 C \epsilon)^{1 / 6}, \quad C=1.1 \text {. }
$$

For small finite $F$, Eq. (2.21) has to be replaced by

$$
E_{r}+U_{\mathrm{eff}}\left(\xi_{0}+\Delta \xi\right)=U_{\mathrm{eff}}\left(\xi_{m}\right)
$$

where $\xi_{0}$ and $\xi_{m}$ are the minimum and maximum of the potential $U_{\mathrm{eff}}(\xi)$. Designating

$$
v \equiv V_{1} \epsilon^{1 / 6}, \quad f \equiv F_{1} \epsilon^{5 / 3}|\ln \epsilon|^{-1},
$$

then we obtain

$$
F_{1}=\frac{3}{8 \pi}\left(V_{1}^{4}-\frac{4 C}{V_{1}^{2}}\right)
$$

and

$$
\gamma=\frac{\pi f}{4 v}=\frac{\pi F_{1}}{4 V_{1}} \epsilon^{3 / 2}|\ln \epsilon|^{-1}
$$

For $f<\epsilon, \epsilon^{1 / 6}<<v<<1$, the energy of the reflected fluxon may be neglected, and we obtain

$$
U\left(\xi_{0}+\Delta \xi\right)=U\left(\xi_{m}\right),
$$

hence

$$
v=\left[\frac{4 \pi f}{\epsilon}\left|\ln \left[\frac{f}{\epsilon}\right]\right|\right]^{1 / 4}, \quad \gamma=\epsilon^{1 / 4} f^{3 / 4}\left|\ln \frac{f}{\epsilon}\right|^{-1 / 4}
$$

In the case of $f \sim \gamma \sim \epsilon, v \sim 1$, it is necessary to insert into (2.22) the full relativistic expression for $\Delta \xi$. The results obtained numerically are shown in Fig. 6.

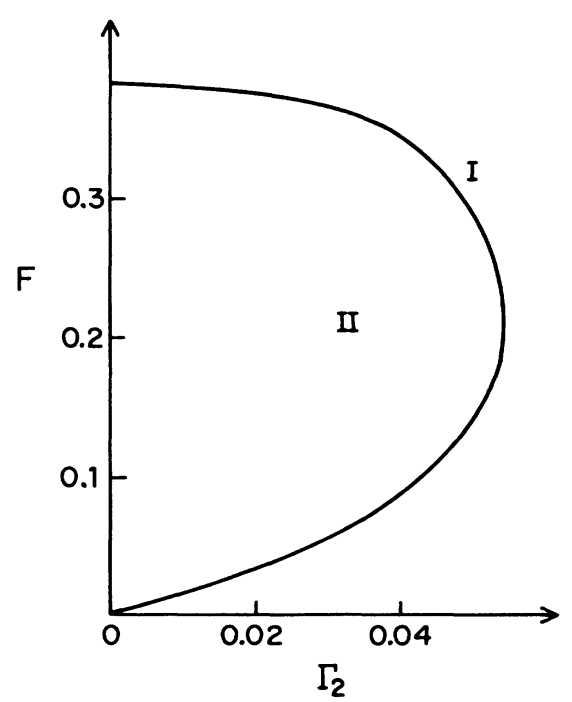

FIG. 6. Interaction regimes in the case of the relativistic incoming fluxon and the attractive inhomogeneity (I-depinning, and II-exchange).

\section{THE COLLISION BETWEEN THE FLUXONS WITH OPPOSITE POLARITIES}

In the case when the free fluxon runs into the pinned antifluxon, the analysis proves to be simpler than in the case of like polarities. The difference is that fluxons with opposite polarities pass through each other during the collision. This implies that the interaction between the fluxons is strong, and one may neglect the relatively weak interaction with the inhomogeneity.

First of all, it is easy to find the threshold for annihilation of the free fluxon with the pinned antifluxon. The annihilation threshold for the free fluxon-antifluxon pair has been found in Refs. 7 and 8. The analysis developed in these works was based on equating the dissipative losses for the pair with the zero velocities of the fluxons at infinity to the net kinetic energy of the actual pair. In the present case, the only difference is that the pinned fluxon has no kinetic energy, consequently, the velocity of the free fluxon corresponding to the annihilation threshold is $\sqrt{2}$ times larger than in the case of the annihilation of the free pair. Using the results of Refs. 7 and 8 , one can find that the annihilation takes place in the region

$$
f \leq f_{\text {ann }} \equiv 2(2 \gamma)^{3 / 2} \text {. }
$$

If $f$ exceeds the value $f_{\text {ann }}$, the outcome of the collision may be either the elastic collision or the depinning. To find a boundary between them in the parametric space, we can use the expression (2.17) for the collision-induced shift of the fluxons. The distance by which each fluxon is shifted does not depend on their relative polarity, ${ }^{4}$ but the direction of the shift depends on it. When we considered the collision of the unipolar fluxons, the moving one stopped and the quiescent one got moving, so that, effectively, one could say that the collision gave rise to a shift of the quiescent fluxon backward relative to the direction of motion of the free one. In the present case, the attraction to the moving antifluxon shifts the quiescent fluxon forward. Considering the position of the shifted fluxon in the effective potential (1.5), one can readily see that it will be depinned if the shift exceeds the distance $l$ between the maximum and the minimum of the potential. If one assumes $f<|\epsilon|$, it is straightforward to find

$$
l \approx \frac{1}{2} \ln (|\epsilon| / 2 \pi f) .
$$

Comparing (3.2) and (2.17), we conclude that the depinning $(l<\Delta \xi)$ takes place provided

$$
v^{4}<32 \pi f / \epsilon
$$

Finally, making use of (1.4) to express $v$ in terms of $f$, we conclude that the depinning condition (3.3) amounts to the inequality

$$
f \leq f_{\text {dep }} \equiv \frac{16}{\pi}\left(\frac{2 \gamma^{4}}{|\epsilon|}\right)^{1 / 3} .
$$

If $f$ exceeds the value $f_{\text {dep }}$, the collision will be basically elastic. 
Comparing (3.1) and (3.4) reveals that the inequality $f_{\text {ann }} \ll f_{\text {dep }}$ always holds. Thus, we expect that in the case of the opposite polarities the annihilation is changed by the depinning and then by the elastic interaction with the increase of the driving force $f$.

Of course, the threshold values (3.2) and (3.4) are meaningful when they do not exceed the critical value (1.7) at which the pinned state disappears. If $f_{\text {ann }}>f_{\mathrm{cr}}$, the collision always results in the annihilation, and if $f_{\text {ann }}<f_{\text {cr }}<f_{\text {dep }}$, the elastic interaction never takes place.

\section{CONCLUSION}

The problems considered in the present work can be generalized in several directions. First of all, one can consider another type of the local inhomogeneity that detains the pinned fluxon. Although the microshorts and microresistors are of most interest in the theory of the LJJ's, the inhomogeneity induced by Abrikosov's vortex crossing the junction is of certain interest too. This inhomogeneity is described by the $\operatorname{term}^{9} \epsilon \delta^{\prime}(x)$ on the righthand side of the perturbed SG equation. The interaction between the free fluxon and the one pinned by Abrikosov's vortex has also been analyzed by the present authors, ${ }^{10}$ but this analysis does not give principally new results.

A more general type of the local inhomogeneity than that on the right-hand side of (1.1) corresponds to a charged impurity in a commensurate charge-densitywave system. In a general case, this inhomogeneity is described by the term ${ }^{11} \epsilon \sin \left[M^{-1}(\phi+\theta)\right]$, where $M$ is the integer commensurability index, and $\theta$ is an arbitrary constant characterizing the location of the impurity relative to the ionic lattice. The important difference of this generalized inhomogeneity from the one considered in the present paper is that the effective fluxon's potential corresponding to it may have four equilibrium positions. ${ }^{11}$

It would be interesting to consider the interaction between the free and pinned fluxons outside the framework of the perturbation theory, when the parameters in (1.1) are not small. In this case, one may expect new types of the interaction, which were absent in the case comprised by the perturbation theory. For instance, if the polarities of the fluxons are opposite, the "recharging" process could be possible (similar to the exchange in the case of the like polarities), i.e., the incoming fluxon becomes pinned, while the pinned one is released. However, the nonperturbative analysis must be essentially based on numerical methods.

Finally, the extension to other models, different from the SG one, could also be worthwhile. A natural problem is to consider the interaction of the free and pinned $2 \pi$ kinks in the damped double SG model with the local inhomogeneity: ${ }^{12}$

$$
\phi_{t t}-\phi_{x x}+\sin \phi=-\gamma \phi_{t}+\alpha \sin (\phi / 2)+\epsilon \delta(x) \sin \phi .
$$

Interaction of the free $2 \pi$-kink with a pinned $4 \pi$-kink could be of interest too. Another model problem is to analyze the collision between the free kink in the dc-driven damped $\phi^{4}$ model with the antikink pinned by a local inhomogeneity (in the $\phi^{4}$ model, only the collisions between kinks with opposite polarities are possible).

\section{ACKNOWLEDGMENT}

A. A. N. acknowledges the support of the Barecha Foundation.
*Present address: School of Mathematical Sciences, Tel Aviv University, Ramat Aviv 69978, Israel.

$\dagger$ The author to whom correspondence should be addressed.

${ }^{1}$ D. W. McLaughlin and A. C. Scott, Phys. Rev. A 18, 1652 (1978).

${ }^{2}$ H. Akoh, S. Sakai, A. Yagi, and H. Hayakawa, IEEE Trans. Magn. 21, 737 (1985).

${ }^{3}$ I. L. Serpuchenko and A. V. Ustinov, Zh. Eksp. Teor. Fiz. 46, 435 (1987) [JETP Lett. 46, 549 (1987)].

${ }^{4}$ J. Rubinstein, J. Math. Phys. 11, 258 (1970).

${ }^{5}$ Yu. S. Kivshar, B. A. Malomed, and A. A. Nepomnyashchy, Zh. Eksp. Teor. Fiz. 94, 356 (1988) [Sov. Phys. JETP 67, 850 (1988)].

${ }^{6}$ V E. Zakharov, S. V. Manakov, S. P. Novikov, and L. P. Pi- taevsky, Soliton Theory: Inverse Scattering problem method (Nauka, Moscow, 1980) [English translation: Consultants Bureau, New York, 1984].

${ }^{7}$ B. A. Malomed, Physica D 15, 385 (1985).

${ }^{8}$ N. F. Pedersen, M. R. Samuelsen, and D. Welner, Phys. Rev. B 30, 4057 (1984).

${ }^{9}$ L. G. Aslamazov and E. V. Gurovich, Zh. Eksp. Teor. Fiz. 40, 22 (1984) [JETP Lett. 40, 746 (1984)].

${ }^{10}$ B. A. Malomed and A. A. Nepomnyashchy (unpublished).

${ }^{11}$ B. A. Malomed and A. A. Nepomnyashchy, J. Phys. Condens. Matter 3, 693 (1991).

${ }^{12}$ Yu. S. Kivshar and B. A. Malomed, Phys. Lett. A 122, 245 (1987). 